\title{
Prognostic significance of pretreatment controlling nutritional status score in urological cancers: a systematic review and meta-analysis
}

\author{
Xinhao Niu, Zhe Zhu and Juan Bao* (10)
}

\begin{abstract}
Background: Controlling Nutritional Status (CONUT) score is a novel nutrition-based biomarker that has been reported for predicting survival in various cancers. However, the relationship between CONUT score and prognosis of urological cancers remains unclear. Hence, we performed this meta-analysis to evaluate the prognostic significance of CONUT score for patients with urological cancers.

Methods: PubMed, Embase, the Cochrane Library and National Knowledge Infrastructure (CNKI) were systematically searched up to October 2020. The pooled hazard ratios (HRs) with 95\% confidence intervals (Cls) were calculated to evaluate the association of CONUT score with overall survival (OS), cancer-specific survival (CSS) and recurrence/disease/progress-free survival (RFS/DFS/PFS) in urological cancers.
\end{abstract}

Results: A total of 12 articles with 13 studies were included in the analysis. Pooled results indicated that increased CONUT score predicted poor OS (HR: 1.78, 95\% Cl 1.51-2.09, p<0.001), CSS (HR: 2.14, 95\% Cl 1.55-2.97, p<0.001) and RFS/DFS/PFS (HR: 1.57, 95\% Cl 1.35-1.84, $\mathrm{p}<0.001$ ). Subgroup analysis by cancer type revealed that high CONUT score associated with worse OS in renal cell carcinoma (RCC) and urothelial cancer (UC) (HR: 3.05, 95\% Cl 2.07-4.50, $\mathrm{p}<0.001$; HR: $1.58,95 \% \mathrm{Cl} 1.32-1.89, \mathrm{p}<0.001$ ). Similar results could be found in CSS (RCC HR: $2.67,95 \% \mathrm{Cl} 1.87-3.81$, $p<0.011$; UC HR: $1.68,95 \% \mathrm{Cl} 1.09-2.59, \mathrm{p}=0.011)$ and in RFS/DFS/PFS (RCC HR: 1.96, 95\% Cl 1.44-2.66, $p<0.001$; UC HR: $1.42,95 \% \mathrm{Cl} 1.18-1.71, \mathrm{p}<0.001)$.

Conclusions: These results illustrated that the high CONUT score may predict worse survival for patients suffering from urological cancers. Therefore, the CONUT score may represent an effective prognostic indicator in urological cancers.

Keywords: CONUT score, Urological cancers, Meta-analysis, Prognosis

\section{Background}

Urological cancers, mostly containing renal cell carcinoma (RCC), prostate cancer (PC) and urothelial cancer (UC), are the major public health problem around the world [1]. According to global cancer statistics, urological cancers accounted for greater than $32 \%$ of all kinds of malignant tumors in 2019 [2]. RCC is a common

*Correspondence: bj901120@163.com

Department of Urinary Surgery, Shanghai Public Health Clinical Center,

Fudan University, No. 2901 Caolang Road, 201508 Shanghai, China malignant tumor of the urinary system, with more than 70,000 new cases and 10,000 deaths in 2020 [3]. During the latest cancer statistics, $\mathrm{PC}$ is predicted to be the third most commonly diagnosed cancer in the world, with more than one million new cases annually [3]. UC, mainly comprising bladder cancer $(\mathrm{BC})$ and upper tract urinary cancer (UTUC), is estimated to cause over 18,000 deaths in the United States each year [4]. Despite the progress of the therapies and techniques for urological cancers including chemotherapy and molecular targeted therapy, the clinical prognosis of urological cancers remains not 
significantly increase in the past two decades, partly due to recurrence and metastasis [5]. Based on the expected survival time of patients to develop treatment plan are important for improving the cure rate of urological cancers. At present, the treatment of urological cancers is mainly based on pathological stage. Nevertheless, the current stage system is not enough to support the choice of treatment and the evaluation of prognosis of urological cancers [6]. Therefore, it is critical to explore a new prognostic biomarker to guide the treatment of urological cancers.

Accumulating evidence demonstrates that host nutritional status plays an important role in progression of cancers and survival of cancer patients [7]. Several nutritional assessment biomarkers such as prognostic nutritional index (PNI) [8], Glasgow prognostic score (GPS) [9], modified Glasgow prognostic score (mGPS) [9], albumin-to-globulin ratio (AGR) [10] and serum albumin [11] have been confirmed as prognostic factors in urological cancers. The CONUT score, calculated from three peripheral blood parameters (total lymphocyte count, serum albumin concentration and total cholesterol level, Table 1), is a newly proposed scoring system to evaluate nutritional status of patients. The three peripheral blood parameters were convenient blood parameters and easy to be acquired during routinely clinical practice. Furthermore, compared to the biomarkers (PNI, GPS, mGPS, AGR and serum albumin) which were determined from only one or two types of serum markers, the CONUT score may be able to provide a more comprehensive clinical picture of the balance of host nutritional and immune status since it is derived from up to three blood parameters. High CONUT signifies low levels of lymphocytes, albumin and cholesterol, which is typically associated with the poor nutritional and immune status of patients and it can lead to worse survival. Recently, the prognostic value of the CONUT score has been reported in gastric

Table 1 Definition of CONUT score

\begin{tabular}{lllll}
\hline Parameters & \multicolumn{2}{l}{ CONUT } & & \\
\cline { 2 - 5 } & Normal & Light & Moderate & Severe \\
\hline Serum albumin $(\mathrm{g} / \mathrm{dL})$ & $3.5-4.5$ & $3.0-3.49$ & $2.5-2.99$ & $<2.5$ \\
Sore & 1 & 2 & 4 & 6 \\
$\begin{array}{l}\text { Total lymphocyte (count/ } \\
\left.\quad \text { mm }^{3}\right)\end{array}$ & $\geq 1600$ & $1200-1599$ & $800-1199$ & $<800$ \\
Sore & 0 & 1 & 2 & 3 \\
Total cholesterol (mg/dL) & $>180$ & $140-180$ & $100-139$ & $<100$ \\
Sore & 0 & 1 & 2 & 3 \\
CONUT score (total) & $0-1$ & $2-4$ & $5-8$ & $9-12$ \\
\hline
\end{tabular}

cancer [12], hepatocellular carcinoma [13] and colorectal cancer [14]. Nonetheless, the application of CONUT score as a prognostic indicator in patients with urological cancers remains inconsistent. For example, Miyaka et al. [15] and Takemura et al. [16] considered high CONUT score was not associated with patients' prognosis, while other studies $[17,18]$ suggested CONUT score was an effective prognostic indicator in urological cancers. Thus, we aimed to systematical review the published literatures, and investigate CONUT score as prognosis predictor in urological cancers, so as to provide high-level evidence for this issue.

\section{Materials and methods}

\section{Search strategy and selection criteria}

This work was conducted according to the preferred reporting items for systemic reviews and meta-analysis (PRISMA) [19]. A comprehensive literature search for relevant studies to assess the relationship between the CONUT score and prognosis of urological cancers in PubMed, Embase, the Cochrane Library and National Knowledge Infrastructure (CNKI) databases, published up to October 2020. The used search terms were "controlling nutritional status", "CONUT", "renal cell cancer", "bladder cancer", "prostate cancer", "urothelial cancer", "urological cancer", "prognosis", and "survival".

Two independent authors (XHN and ZZ) reviewed all candidate articles. Literatures were finally included when they met the following criteria: (1) patients were histopathologically diagnosed with urological cancers, including RCC, BC, PC UTUC; (2) reported the relationship between pretreatment CONUT score and overall survival (OS) or cancer-specific survival (CSS) or recurrence/disease/progress-free survival (RFS/DFS/PFS); (3) provided hazard ratio (HR) and $95 \%$ confidence intervals (CI) directly. Studies were excluded when they met the following criteria: (1) letters, reviews, abstract and case reports; (2) lack of accurate data; (3) the cut-off value did not given.

\section{Data extraction and quality assessment}

The following data were extracted by two authors (XHN and ZZ): first author's surname, country of origin, year of publication, number of patients, duration of followup, cancer type and stage, treatment methods, design of study, cut-off value, and survival analysis. The quality of all primary studies were independently assessed in the light of the Newcastle-Ottawa Scale (NOS) [20] by three investigators (XHN, ZZ and JB). Total quality scores were ranged from 0 to 9 and if the final score $>6$, we regarded it to be of high quality. 


\section{Statistical analysis}

HR and 95\% CI were directly extracted from each publication to evaluate the importance of prognostic role of CONUT score for patients with urological cancers. To pool the overall HR with $95 \% \mathrm{CI}$, the HR from the multivariate analysis was extracted from each study. The Cochran $\mathrm{Q}$ and $\mathrm{I}^{2}$ statistical methods were applied to evaluate the heterogeneity among included studies [21]. A fixed-effects model was used to calculate the pooled estimates in the absence of heterogeneity $\left(\mathrm{I}^{2}<50 \%\right.$ or $\mathrm{p}>0.10)$. Otherwise, a random-effects model was applied. According to cancer type, cancer stage, treatment methods, sample size and cut-off value, further subgroup analysis was also conducted. Potential publication bias was evaluated by Begg's test with funnel plots. A p-value $<0.05$ was regarded statistically significant. In addition, a sensitivity analysis was carried out to assess the influence of each individual study on the pooled results by sequentially excluding each study. All analyses were performed by using Stata software version 12.0.

\section{Results}

\section{Characteristics of selected articles}

The selection procedure was presented in a flow diagram (Fig. 1). Finally, 12 articles with 13 studies were included for meta-analysis [15-18, 22-29]. The features of the 13 studies were outlined in Table 2. All included studies were evaluated as high quality according to the NOS (Table 3). Among the studies, 6 focused on RCC, 6 focused on UC and 1 focused on PC. Of six studies on $\mathrm{UC}, 2$ investigated bladder urothelial cancer and 3 investigated UTUC. A total of 6 studies were conducted in China, 4 performed in Japan, 2 from Korean and 1 from Egypt. The sample sizes were ranged from 49 to 1418. In terms of treatment methods, target therapy was investigated in 1 studies and operation was investigated in 11

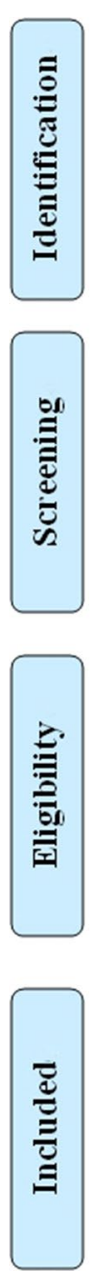

\section{Records identified through} database searching $(n=49)$

\section{Additional records identified} through other sources $(\mathrm{n}=0)$
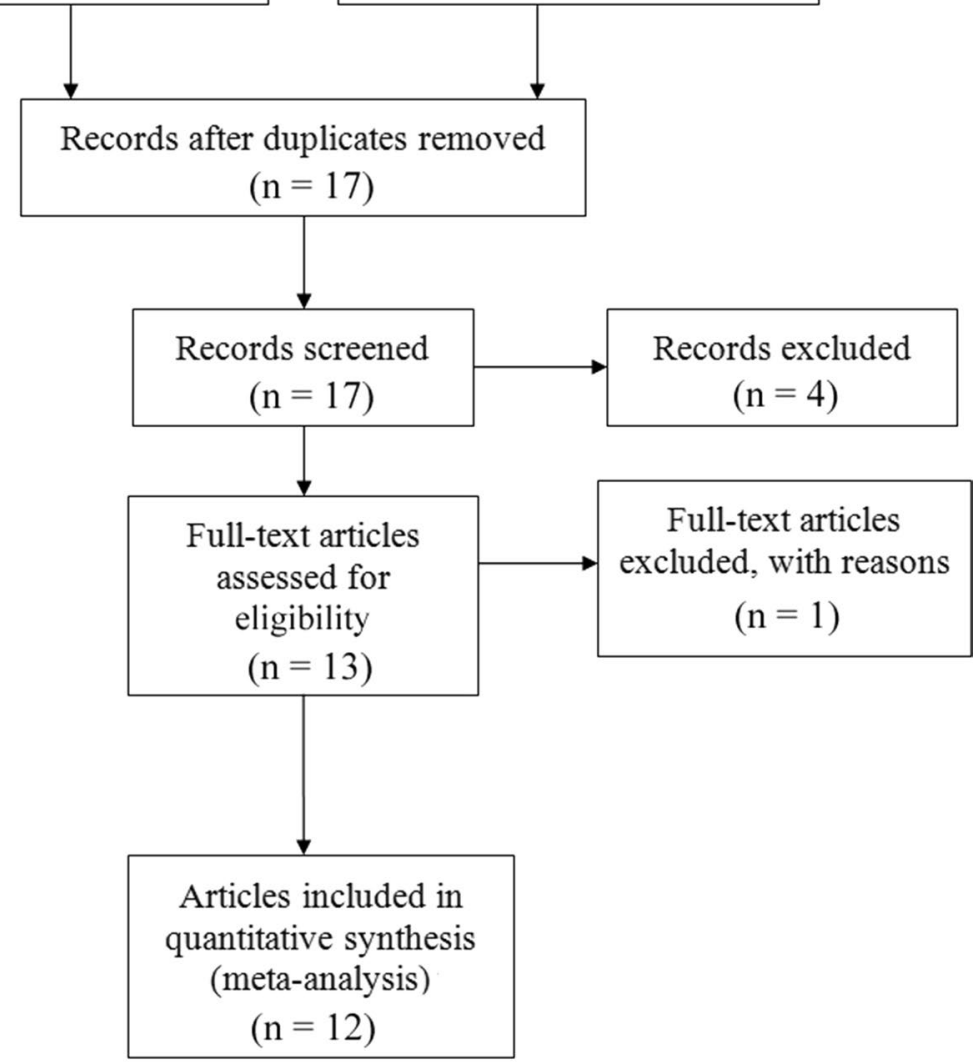

Fig. 1 Flow chart of the meta-analysis 


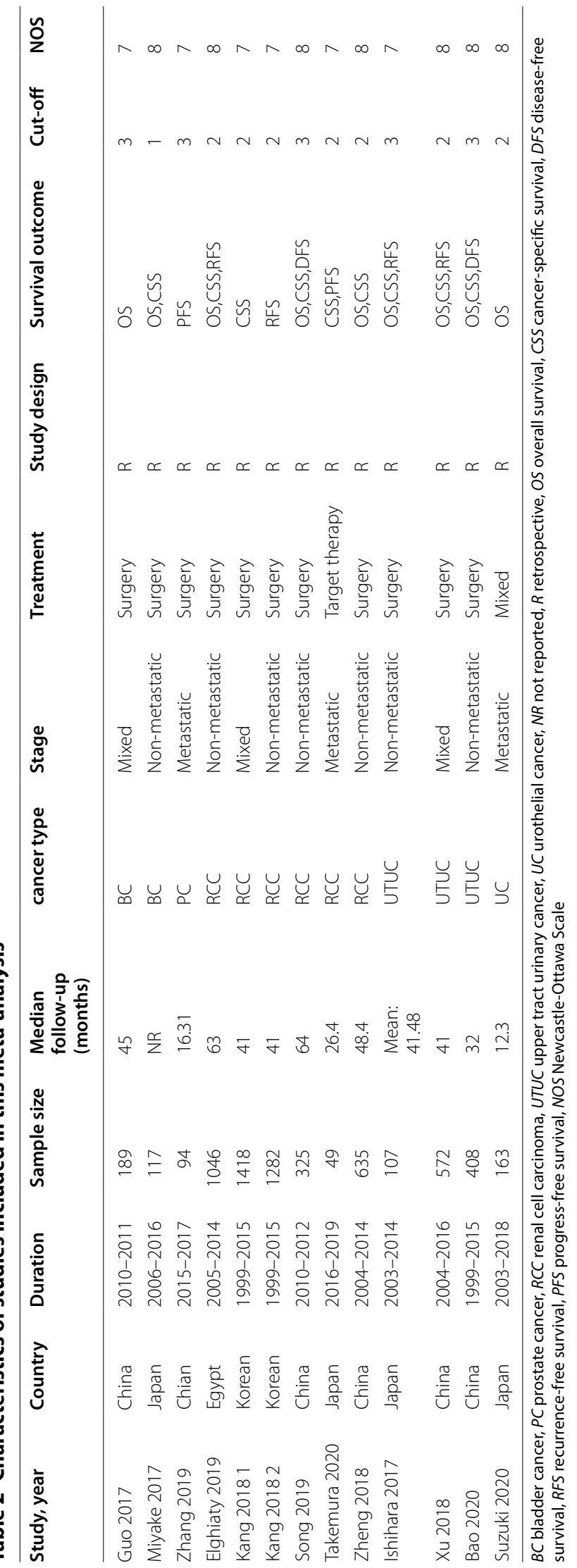


Table 3 Newcastle-Ottawa Scale for quality assessment

\begin{tabular}{|c|c|c|c|c|c|c|c|c|c|}
\hline \multirow[t]{2}{*}{ Studies } & \multicolumn{8}{|c|}{ Quality indicators from Newcastle-Ottawa Scale } & \multirow[t]{2}{*}{ Scores } \\
\hline & 1 & 2 & 3 & 4 & 5 & 6 & 7 & 8 & \\
\hline Guo 2017 & $\star$ & $\star$ & - & $\star$ & $\star \star$ & $\star$ & $\star$ & - & 7 \\
\hline Miyake 2017 & $\star$ & $\star$ & - & $\star$ & $\star \star$ & $\star$ & $\star$ & $\star$ & 8 \\
\hline Zhang 2019 & $\star$ & $\star$ & $\star$ & $\star$ & $\star \star$ & - & - & $\star$ & 7 \\
\hline Elghiaty 2019 & $\star$ & $\star$ & $\star$ & $\star$ & $\star \star$ & - & $\star$ & $\star$ & 8 \\
\hline Kang 20181 & $\star$ & $\star$ & - & - & $\star \star$ & $\star$ & $\star$ & $\star$ & 7 \\
\hline Kang 20182 & $\star$ & $\star$ & - & - & $\star \star$ & $\star$ & $\star$ & $\star$ & 7 \\
\hline Song 2019 & $\star$ & $\star$ & $\star$ & $\star$ & $\star \star$ & - & $\star$ & $\star$ & 8 \\
\hline Takemura 2020 & $\star$ & $\star$ & - & $\star$ & $\star \star$ & - & $\star$ & $\star$ & 7 \\
\hline Zheng 2018 & $\star$ & $\star$ & - & $\star$ & $\star \star$ & $\star$ & $\star$ & $\star$ & 8 \\
\hline Ishihara 2017 & $\star$ & $\star$ & - & $\star$ & $\star \star$ & - & $\star$ & $\star$ & 7 \\
\hline Xu 2018 & $\star$ & $\star$ & - & $\star$ & $\star \star$ & $\star$ & $\star$ & $\star$ & 8 \\
\hline Bao 2020 & $\star$ & $\star$ & $\star$ & $\star$ & $\star \star$ & - & $\star$ & $\star$ & 8 \\
\hline Suzuki 2020 & $\star$ & $\star$ & - & $\star$ & $\star \star$ & $\star$ & $\star$ & $\star$ & 8 \\
\hline
\end{tabular}

1 Representativeness of the exposed cohort, 2 Selection of the non-exposed cohort, 3 Ascertainment of exposure, 4 Outcome of interest not present at start of study, 5 Control for important factor or additional factor, 6 Assessment of outcome, 7 Follow-up long enough for outcomes to occur, 8 Adequacy of follow up of cohorts

studies. Among the included studies, 9 evaluated OS, 9 evaluated CSS, 8 evaluated RFS/DFS/PFS.

\section{The CONUT score and OS in urological cancers}

A total of 9 studies with 3562 patients explored the role of CONUT score on OS. The fixed-effect model was used to calculated the pool results $\left(\mathrm{I}^{2}=45.3 \%, \mathrm{p}=0.067\right)$. As shown in Fig. 2, the pooled data revealed that increased CONUT score was associated with worse OS (HR: 1.78, 95\% CI 1.51-2.09, p<0.001). Subgroup analysis by cancer type for OS indicated that elevated CONUT score can predict poor OS for RCC (HR: 3.05, 95\% CI 2.07$4.50, \mathrm{p}<0.001$ ) and UC (HR: $1.58,95 \%$ CI $1.32-1.89$, $\mathrm{p}<0.001)$. After stratification by cancer stage, the pooled

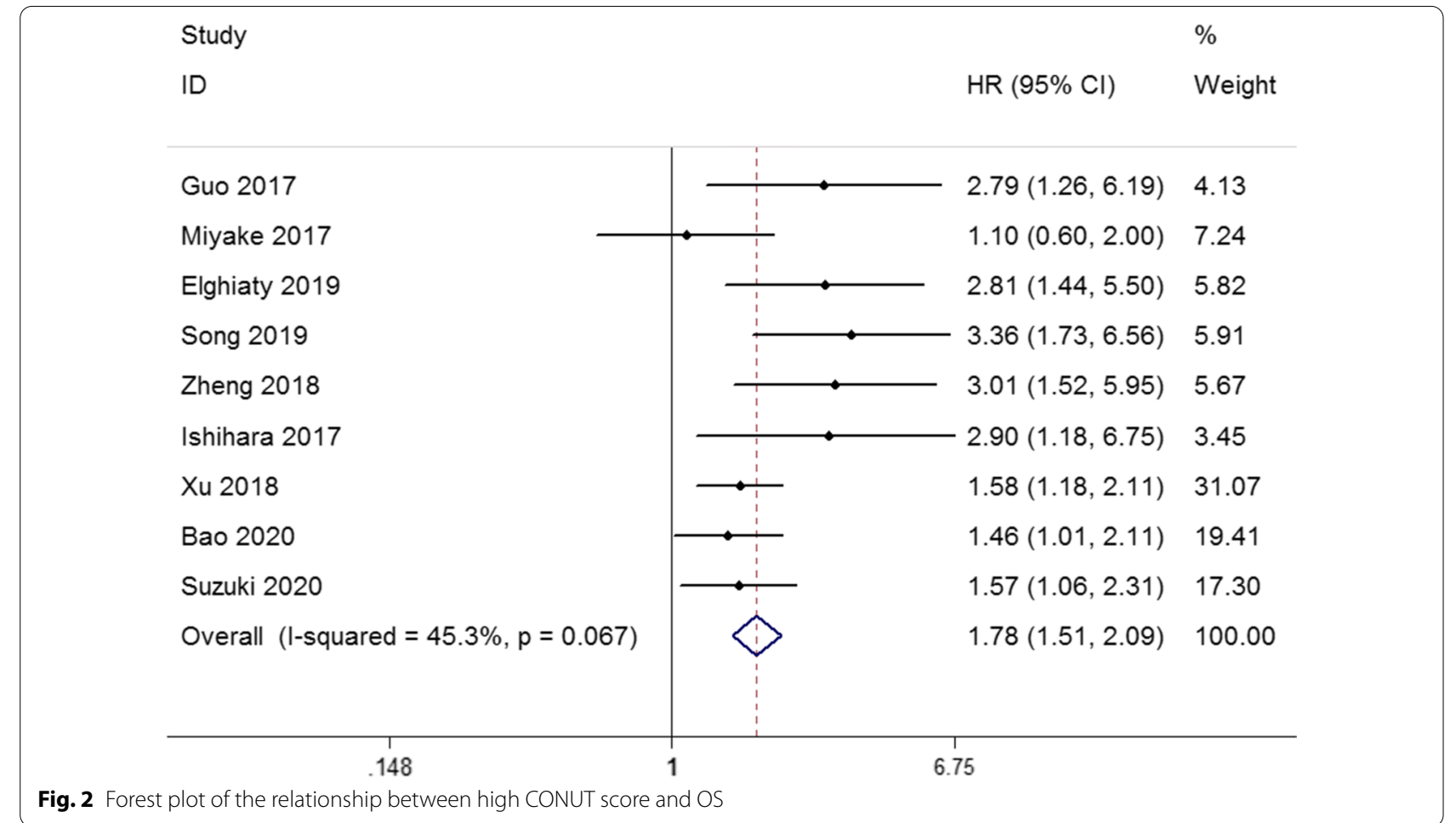


HR was 2.14 (95\% CI $1.45-3.15, \mathrm{p}<0.001)$ in non-metastatic group and $1.69(95 \%$ CI $1.29-2.22, \mathrm{p}<0.001)$ in mixed group. In terms of treatment methods, pretreatment CONUT score could be a negative predictor for OS in patients undergoing surgery (HR: 2.02, 95\% CI $1.53-2.68, \mathrm{p}<0.001)$. In the analysis of sample size, the pooled HR was 1.73 (95\% CI 1.40-2.12, $\mathrm{p}<0.001)$ for sample size $>400$ and 2.02 (95\% CI 1.33-3.07, p < 0.001) for sample size $<400$. In addition, when the cut-off value of CONUT score $=3$ (HR: 2.31, 95\% CI 1.44-3.72, $\mathrm{p}=0.001)$ and CONUT score $\leq 2$ (HR: $1.68,95 \% \mathrm{CI}$ $1.38-2.05, \mathrm{p}<0.001)$, it was also negatively associated with OS. Finally, among Asian populations, high CONUT score could be a negative predictor for OS in urological cancers (HR: 1.73, 95\% CI 1.46-2.04, p<0.001). These results are illustrated in Table 4.

\section{The CONUT score and CSS in urological cancers}

The effect of CONUT score on CSS was evaluated in 4677 patients from 9 studies. A combined analysis demonstrated that high CONUT score were significantly positively correlated with shortened CSS (HR: 2.14, 95\% CI 1.55-2.97, $\mathrm{p}<0.001)$, with moderate heterogeneity identified between studies $\left(\mathrm{I}^{2}=51.6 \%, \mathrm{p}=0.035\right.$; Fig. 3). Subgroup analysis by cancer type for CSS indicated that elevated CONUT score can predict poor CSS for RCC (HR: 2.67, 95\% CI 1.87-3.81, p<0.011) and UC
(HR: 1.68, 95\% CI 1.09-2.59, $\mathrm{p}=0.019)$. After stratification by cancer stage, the pooled HR was 2.45 (95\% CI $1.44-4.17, \mathrm{p}=0.001)$ in non-metastatic group and 1.74 $(95 \%$ CI $1.32-2.31, \mathrm{p}<0.001)$ in mixed group. In terms of treatment methods, preoperation CONUT score could be a negative predictor for CSS (HR: 2.10, 95\% CI $1.51-2.92, \mathrm{p}<0.001)$. In the analysis of sample size, the pooled HR was 1.77 (95\% CI 1.41-2.20, p<0.001) for sample size $>400$ and 2.83 (95\% CI 1.23-6.50, $\mathrm{p}=0.014)$ for sample size $<400$. In addition, when the cut-off value of CONUT score $=3$ (HR: 2.66, 95\% CI 1.13-6.27, $\mathrm{p}=0.026)$ and CONUT score $\leq 2$ (HR: $1.85,95 \% \mathrm{CI}$ $1.45-2.35, \mathrm{p}<0.001)$, it was also negatively associated with CSS. Finally, CONUT score was also an appropriate biomarker to predict worse CSS among Asian populations (HR: 1.82, 95\% CI 1.49-2.23, p<0.001). These results are illustrated in Tables 4, 5 .

The CONUT score and RFS/DFS/PFS in urological cancers As shown in Fig. 4, a total of 8 eligible studies comprising 3883 patients revealed the relationship between CONUT score and RFS/DFS/PFS by fixed-effects model with low heterogeneity $\left(\mathrm{I}^{2}=19.0 \%, \mathrm{p}=0.279\right)$ in urological cancers (HR: $1.57,95 \%$ CI 1.35-1.84, $\mathrm{p}<0.001$ ) especially in RCC (HR: 1.96, 95\% CI 1.44-2.66, $\mathrm{p}<0.001)$ and UC (HR: 1.42, $95 \%$ CI 1.18-1.71, $\mathrm{p}<0.001)$. Subgroup analysis by treatment methods indicated that CONUT score could be a

Table 4 Subgroup analyses of overall survival

\begin{tabular}{|c|c|c|c|c|c|c|}
\hline \multirow[t]{2}{*}{ Subgroup } & \multirow[t]{2}{*}{ No. of studies } & \multirow[t]{2}{*}{ HR $(95 \% \mathrm{Cl})$} & \multirow[t]{2}{*}{$\mathbf{P}$} & \multicolumn{2}{|c|}{ Heterogeneity } & \multirow[t]{2}{*}{ Model } \\
\hline & & & & $\mathrm{I}^{2}(\%)$ & $\mathrm{Ph}$ & \\
\hline \multicolumn{7}{|l|}{ Cancer type } \\
\hline $\mathrm{RCC}$ & 3 & $3.05(2.07-4.50)$ & $<0.001$ & 0.0 & 0.933 & Fixed \\
\hline UC & 6 & $1.58(1.32-1.89)$ & $<0.001$ & 7.3 & 0.370 & Fixed \\
\hline \multicolumn{7}{|l|}{ Cancer stage } \\
\hline Non-metastatic & 6 & $2.14(1.45-3.15)$ & $<0.001$ & 58.1 & 0.036 & Random \\
\hline Metastatic & 1 & $1.57(1.06-2.31)$ & 0.023 & - & - & - \\
\hline Mixed & 2 & $1.69(1.29-2.22)$ & $<0.001$ & 42.2 & 0.189 & Fixed \\
\hline \multicolumn{7}{|l|}{ Treatment } \\
\hline Surgery & 8 & $2.02(1.53-2.68)$ & $<0.001$ & 50.6 & 0.048 & Random \\
\hline Mixed & 1 & $1.57(1.06-2.31)$ & 0.023 & - & - & - \\
\hline \multicolumn{7}{|l|}{ Sample size } \\
\hline$>400$ & 4 & $1.73(1.40-2.12)$ & $<0.001$ & 47.9 & 0.124 & Fixed \\
\hline$<400$ & 5 & $2.02(1.33-3.07)$ & 0.001 & 53.9 & 0.070 & Random \\
\hline \multicolumn{7}{|l|}{ Cuf-off } \\
\hline 3 & 4 & $2.31(1.44-3.72)$ & 0.001 & 54.0 & 0.089 & Random \\
\hline$\leq 2$ & 5 & $1.68(1.38-2.05)$ & $<0.001$ & 45.0 & 0.122 & Fixed \\
\hline \multicolumn{7}{|l|}{ Population } \\
\hline Asian & 8 & $1.73(1.46-2.04)$ & $<0.001$ & 44.9 & 0.08 & Fixed \\
\hline African & 1 & $2.81(1.44-5.50)$ & 0.003 & - & - & - \\
\hline
\end{tabular}


Table 5 Subgroup analyses of cancer-specific survival

\begin{tabular}{|c|c|c|c|c|c|c|}
\hline \multirow[t]{2}{*}{ Subgroup } & \multirow[t]{2}{*}{ NO. of studies } & \multirow[t]{2}{*}{$\mathrm{HR}(95 \% \mathrm{Cl})$} & \multirow[t]{2}{*}{$P$} & \multicolumn{2}{|c|}{ Heterogeneity } & \multirow[t]{2}{*}{ Model } \\
\hline & & & & $\mathrm{I}^{2}(\%)$ & $\mathrm{Ph}$ & \\
\hline \multicolumn{7}{|l|}{ Cancer type } \\
\hline $\mathrm{RCC}$ & 5 & $2.67(1.87-3.81)$ & $<0.001$ & 0.0 & 0.453 & Fixed \\
\hline UC & 4 & $1.68(1.09-2.59)$ & 0.019 & 59.5 & 0.060 & Random \\
\hline \multicolumn{7}{|l|}{ Cancer stage } \\
\hline Non-metastatic & 6 & $2.45(1.44-4.17)$ & 0.001 & 66.4 & 0.011 & Random \\
\hline Metastatic & 1 & $5.96(0.67-53.01)$ & 0.109 & - & - & - \\
\hline Mixed & 2 & $1.74(1.32-2.31)$ & $<0.001$ & 0.0 & 0.721 & Fixed \\
\hline \multicolumn{7}{|l|}{ Treatment } \\
\hline Surgery & 8 & $2.10(1.51-2.92)$ & $<0.001$ & 54.7 & 0.031 & Random \\
\hline Target therapy & 1 & $5.96(0.67-53.01)$ & 0.109 & - & - & - \\
\hline \multicolumn{7}{|l|}{ Sample size } \\
\hline$>400$ & 5 & $1.77(1.41-2.20)$ & $<0.001$ & 38.2 & 0.166 & Fixed \\
\hline$<400$ & 4 & $2.83(1.23-6.50)$ & 0.014 & 64.6 & 0.037 & Random \\
\hline \multicolumn{7}{|l|}{ Cuf-off } \\
\hline 3 & 3 & $2.66(1.13-6.27)$ & 0.026 & 77.4 & 0.012 & Random \\
\hline$\leq 2$ & 6 & $1.85(1.45-2.35)$ & $<0.001$ & 34.4 & 0.178 & Fixed \\
\hline \multicolumn{7}{|l|}{ Population } \\
\hline Asian & 8 & $1.82(1.49-2.23)$ & $<0.001$ & 48.5 & 0.059 & Fixed \\
\hline African & 1 & $4.66(1.62-13.39)$ & 0.004 & - & - & - \\
\hline
\end{tabular}

\section{Study}

ID
$\%$

$\operatorname{HR}(95 \% \mathrm{Cl}) \quad$ Weight

\section{Miyake 2017}

Elghiaty 2019

Kang 20181

Song 2019

Takemura 2020

Zheng 2018

Ishihara 2017

Xu 2018

Bao 2020

Overall $(\mathrm{I}$-squared $=51.6 \%, p=0.035)$

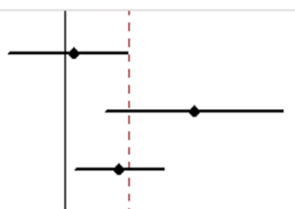

$1.10(0.50,2.10) \quad 11.30$

$4.66(1.63,13.39) \quad 6.92$

$1.89(1.12,3.20) \quad 15.11$

$3.34(1.59,6.98) \quad 10.92$

$5.96(0.67,53.01) \quad 2.05$

$3.00(1.29,6.98) \quad 9.33$

$5.44(1.95,14.80) 7.32$

$1.69(1.21,2.34) \quad 19.84$

$1.34(0.86,2.07) \quad 17.21$

$2.14(1.55,2.97) \quad 100.00$

Fig. 3 Forest plot of the relationship between high CONUT score and CSS 
Table 6 Subgroup analyses of recurrence/disease/progress-free survival

\begin{tabular}{|c|c|c|c|c|c|c|}
\hline \multirow[t]{2}{*}{ Subgroup } & \multirow[t]{2}{*}{ NO. of studies } & \multirow[t]{2}{*}{$\mathrm{HR}(95 \% \mathrm{Cl})$} & \multirow[t]{2}{*}{$\mathbf{P}$} & \multicolumn{2}{|c|}{ Heterogeneity } & \multirow[t]{2}{*}{ Model } \\
\hline & & & & $I^{2}(\%)$ & $\mathrm{Ph}$ & \\
\hline \multicolumn{7}{|l|}{ Cancer type } \\
\hline $\mathrm{RCC}$ & 4 & $1.96(1.44-2.66)$ & $<0.001$ & 0.0 & 0.636 & Fixed \\
\hline UC & 3 & $1.42(1.18-1.71)$ & $<0.001$ & 0.0 & 0.483 & Fixed \\
\hline \multicolumn{7}{|l|}{ Cancer stage } \\
\hline Non-metastatic & 5 & $1.61(1.32-1.97)$ & $<0.001$ & 29.2 & 0.227 & Fixed \\
\hline Metastatic & 2 & $2.65(1.19-5.90)$ & 0.017 & 0.0 & 0.372 & Fixed \\
\hline Mixed & 1 & $1.43(1.10-1.86)$ & 0.008 & - & - & - \\
\hline \multicolumn{7}{|l|}{ Treatment } \\
\hline Surgery & 7 & $1.57(1.34-1.84)$ & $<0.001$ & 29.6 & 0.202 & Fixed \\
\hline Target therapy & 1 & $1.91(0.65-5.61)$ & 0.239 & - & - & - \\
\hline \multicolumn{7}{|l|}{ Sample size } \\
\hline$>400$ & 4 & $1.49(1.25-1.77)$ & $<0.001$ & 36.5 & 0.193 & Fixed \\
\hline$<400$ & 4 & $2.12(1.43-3.15)$ & $<0.001$ & 0.0 & 0.716 & Fixed \\
\hline \multicolumn{7}{|l|}{ Cuf-off } \\
\hline 3 & 4 & $1.54(1.22-1.94)$ & $<0.001$ & 36.6 & 0.192 & Fixed \\
\hline$\leq 2$ & 4 & $1.61(1.30-1.99)$ & $<0.001$ & 21.9 & 0.279 & Fixed \\
\hline \multicolumn{7}{|l|}{ Population } \\
\hline Asian & 7 & $1.53(1.30-1.79)$ & $<0.001$ & 0.0 & 0.487 & Fixed \\
\hline African & 1 & $3.09(1.45-6.59)$ & 0.003 & - & - & - \\
\hline
\end{tabular}

\section{Study}

ID

\section{Zhang 2019}

Elghiaty 2019

Kang 20182

Song 2019

Takemura 2020

Ishihara 2017

Xu 2018

Bao 2020

Overall $(\mathrm{I}$-squared $=19.0 \%, \mathrm{p}=0.279)$
$\mathrm{HR}(95 \% \mathrm{Cl})$

Weight

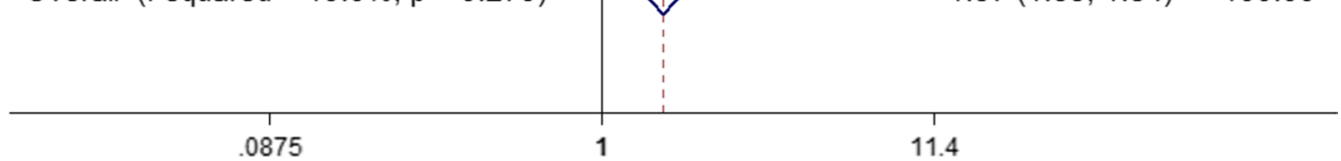

Fig. 4 Forest plot of the relationship between high CONUT score and RFS/DFS/PFS 
negative predictor for RFS/DFS/PFS in patients undergoing surgery (HR: $1.57,95 \%$ CI $1.34-1.84, \mathrm{p}<0.001$ ). In terms of cancer stage the pooled HR was 1.61 (95\% CI $1.32-1.97, \mathrm{p}<0.001)$ in non-metastatic group and 2.65 (95\% CI 1.19-5.90, $\mathrm{p}=0.017)$ in metastatic group. After stratification by sample size, the pooled HR was 1.49 (95\% CI 1.25-1.77, $\mathrm{p}<0.001$ ) in sample size $>400$ group and 2.12 (95\% CI 1.43-3.15, p<0.001) in sample size $<400$ group. Moreover, when the cut-off value of CONUT score $=3$ (HR: $1.54,95 \%$ CI 1.22-1.94, $\mathrm{p}<0.001$ ) and CONUT score $\leq 2$ (HR: $1.61,95 \%$ CI $1.30-1.99, \mathrm{p}<0.001$ ), it was also negatively associated with RFS/DFS/PFS. Finally, high CONUT score was also associated with poor RFS/ DFS/PFS among Asian populations (HR: 1.53, 95\% CI $1.30-1.79, \mathrm{p}<0.001)$. These results are shown in Table 6.

\section{Publication bias and sensitivity analyses}

The funnel plots of Bgger's test were displayed in Fig. 5. Bgger's test revealed that no significant publication bias in this meta-analysis about CONUT score and OS (Fig. 5a, $\mathrm{p}=0.348$ ), CSS (Fig. 5b, $\mathrm{P}=0.076$ ) and RFS/ DFS $/$ PFS (Fig. $5 c, p=0.108$ ). By estimating the potential impact of individual studies on the pooled data, sensitivity analysis was carried out. It was obvious that, pooled HR was not remarkably altered when each single study was removed in turn (Fig. 6a: OS, Fig. 6b: CSS, Fig. 6c: RFS/DFS/PFS).

\section{Discussion}

According to our best knowledge, this is the first meta-analysis which systemically investigated the relationship between CONUT score and the prognosis of urological cancers patients. This meta-analysis of 13 studies demonstrated that high CONUT score predicted poor OS, CSS and RFS/DFS/PFS in urological cancers. A stratified analysis demonstrated that high CONUT score were significantly correlated with decreased OS, CSS and RFS/DFS/PFS, irrespective of the cancer type, cancer stage, treatment methods, sample size and cut-off value. Our results gave the information that pretreatment CONUT score may represent an independent prognostic indicator in patient with urological cancers. Urological cancers contain RCC, PC and UC. RCC is the 13th most common cause of cancer-related mortality worldwide [30]. It is utmostly important to identify the prognostic factors in RCC. Our results confirmed that high COUNT score was associated with worse OS, CSS and RFS/DFS/PFS and CONUT score was an effective prognostic biomarker for RCC. UC, mostly comprising BC and UTUC, have a poor prognosis due to the distant metastasis or postoperative recurrence [31]. To investigate an appropriate prognostic marker is urgent. The subgroup analysis in this study showed that high CONUT score was a prognostic marker for worse OS in UC. Similarly, high CONUT score was also negatively correlated with CSS and RFS/DFS/PFS. Among the included studies in this meta-analysis, only one article investigated the relationship between CONUT score and PC. In that study, the researchers assessed the PFS of CONUT score in PC and considered high CONUT score could predict poor PFS in PC.

The biological mechanism regarding the association between the CONUT score and prognosis is still unclear. However, there are several reasons to explain why a high CONUT score is associated with poor outcomes in urological cancers. High CONUT score are closely related to low levels of serum albumin, cholesterol and lymphocytes. Serum albumin is a major indicator of nutritional status and has been proved associated with worse prognosis in urological cancers [11]. The decreased level of albumin correlated with the production of pro-inflammatory cytokines, such as interleukin-6, a cytokine associated with progression of cancers [32]. Cholesterol is an essential lipid for maintaining cellular, decreased level of cholesterol means a loss of cholesterol from the membrane of cells, which affects the ability of immunocompetent cells to fight against cancer cells [33, 34]. Lymphocytes can show an antitumor role through the induction of cytotoxic cell death [35], and perioperative lymphopenia was reported to be associated with inferior prognosis in cancers [36]. Thus, high CONUT score is typically associated with the invasion and metastasis of tumour cells and it can lead to poor survival.

This meta-analysis had several limitations. First, all enrolled studies were retrospective design, which might contain a potential selection bias. Second, most included studies were performed in Asian countries, therefore it remains unknown whether our results can be applied to Western populations. Third, the cut-off values were inconsistent among the studies. The cutoffs in enrolled studies didn't reach the standard point. Although, according to CONUT $=3$ in the subgroup analysis, we pooled the results, bias may exist because the use of diverse cut-off values caused different conclusions in the same article. Fourth, relevant studies were too few in some subgroup analysis such as only one study investigated the outcome of CONUT score in PC. Moreover, moderate heterogeneity existed in the analysis of CSS. Finally, high CONUT score may have been affected not only by aggressive tumor but also by 


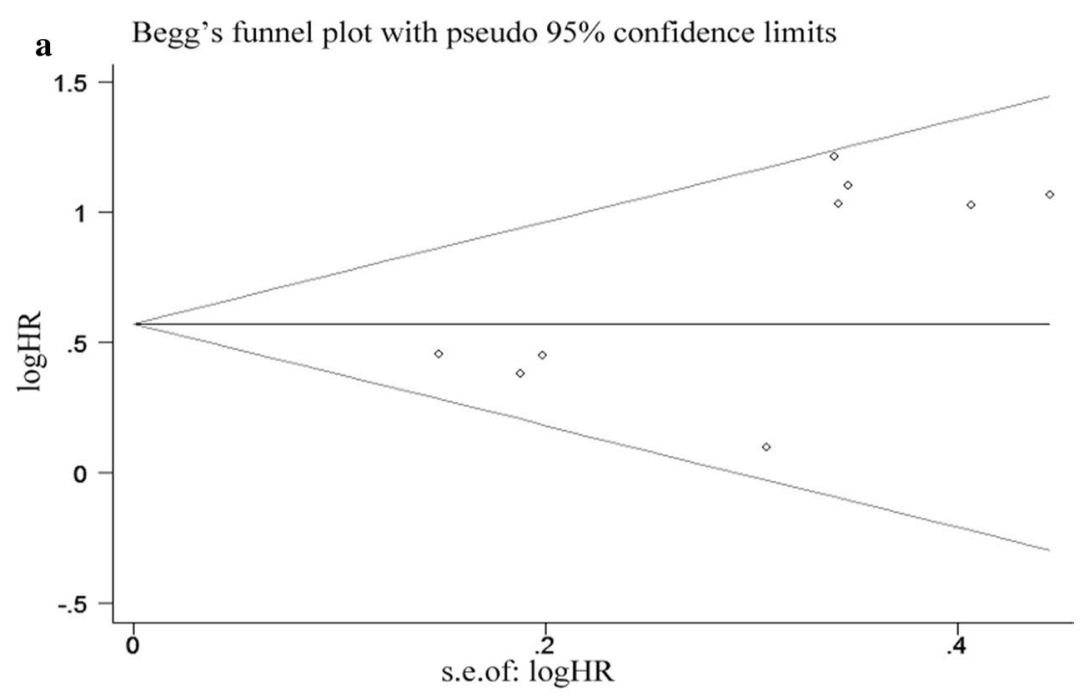

b Begg's funnel plot with pseudo $95 \%$ confidence limits

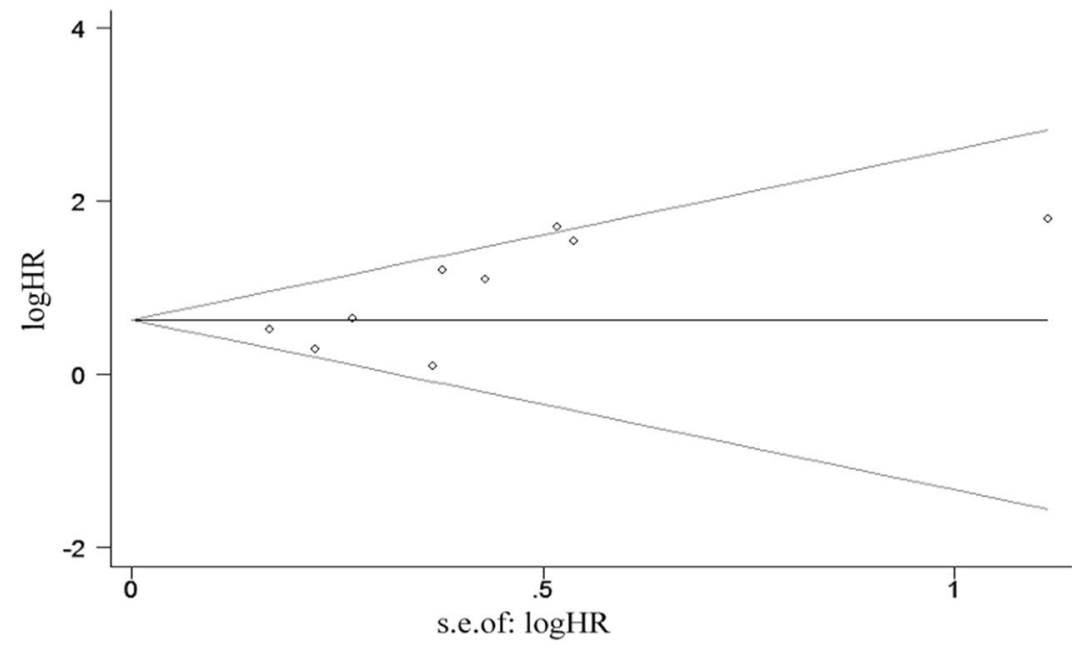

c Begg's funnel plot with pseudo $95 \%$ confidence limits

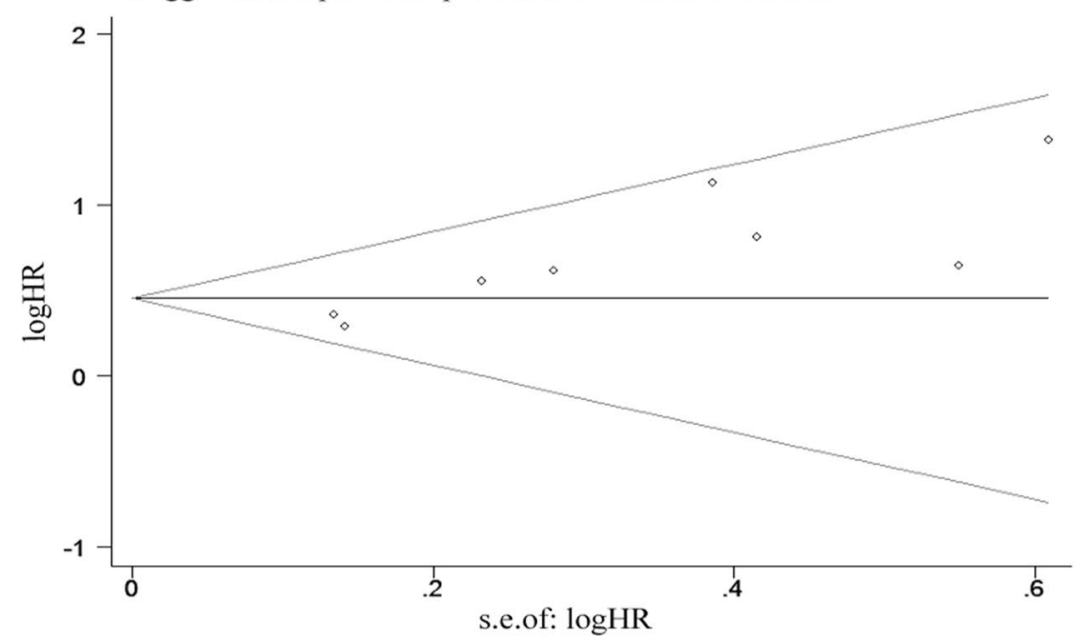

Fig. 5 Begg's publication bias funnel plots for the correlation of high CONUT score with OS (a); with CSS (b); with RFS/DFS/PFS (c) 


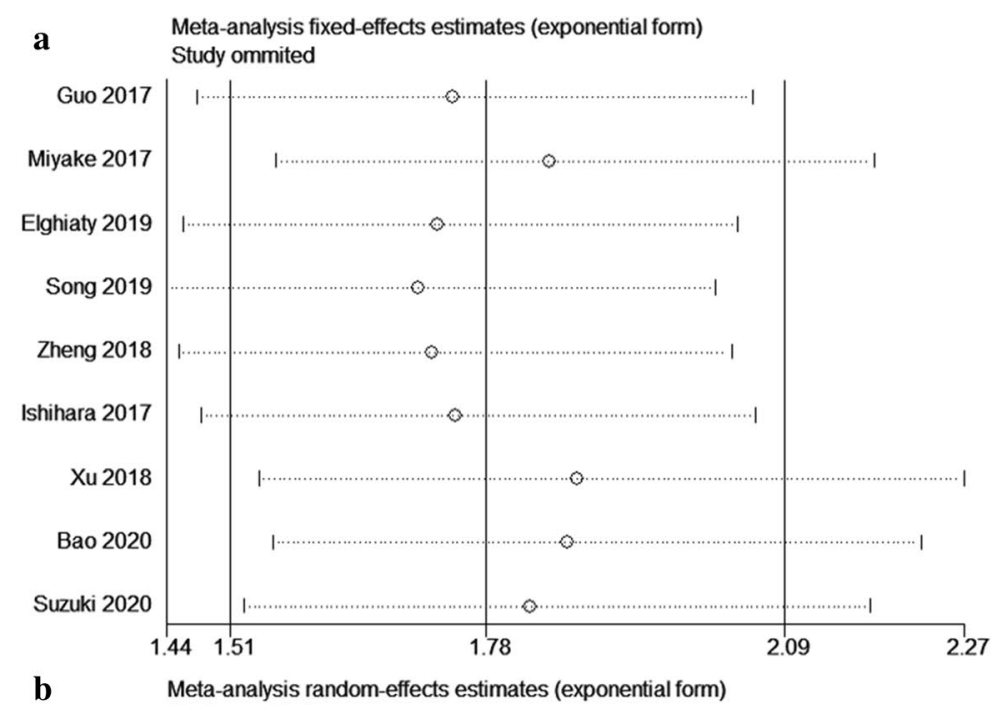

b Meta-analysis random-effects estimates (exponential form)

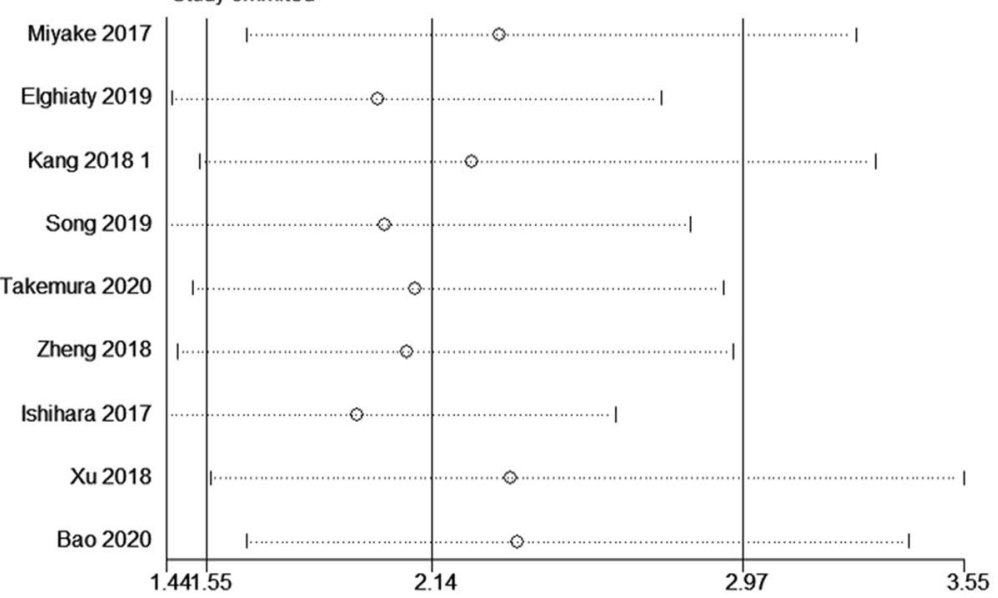

c Meta-analysis fixed-effects estimates (exponential form)

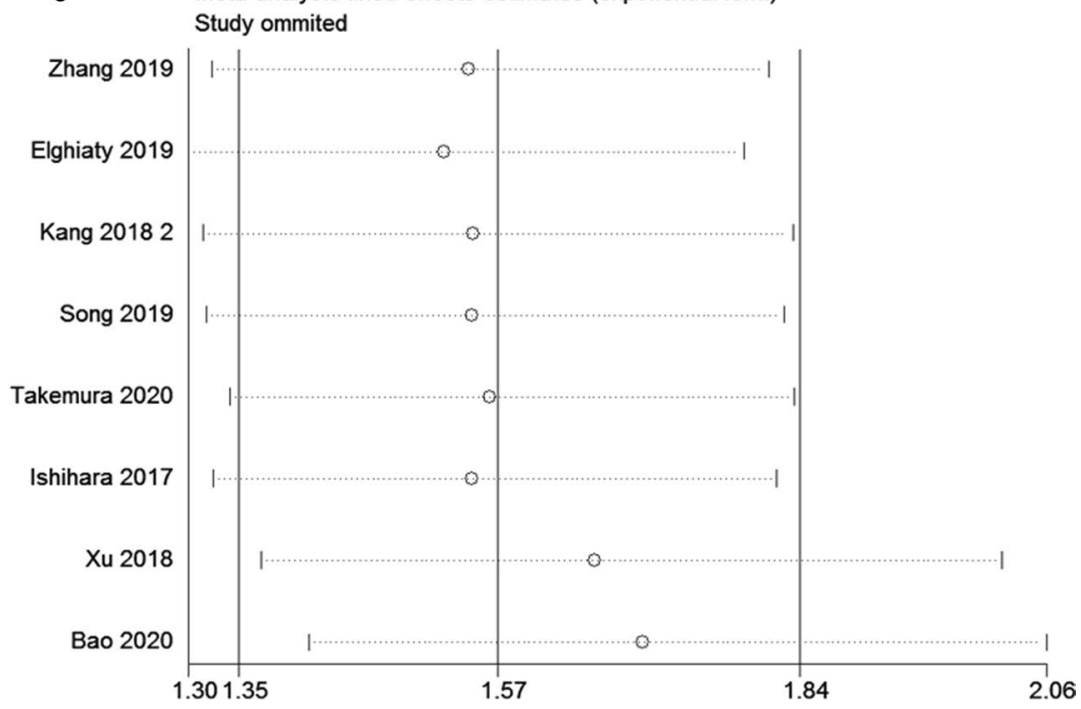

Fig. 6 Sensitivity analysis for the correlation of high CONUT score with OS (a); with CSS (b); with RFS/DFS/PFS (c) 
old age and underlying disease. These articles did not adequately adjust for these confounding factors.

\section{Conclusions}

Our results display that pretreatment CONUT score represents an independent predictive factor for OS, CSS and RFS/DFS/PFS, and the CONUT score may represent an effective prognostic indicator in urological cancers.

\begin{abstract}
Abbreviations
CONUT: Controlling nutritional status; CNKI: National knowledge infrastructure; HR: Hazard ratio; Cl: Confidence interval; BC: Bladder cancer; PC: Prostate cancer; RCC: Renal cell carcinoma; UTUC: Upper tract urinary cancer; UC: Urothelial cancer; OS: Overall survival; CSS: Cancer-specific survival; DFS: Disease-free survival; RFS: Recurrence-free survival; PFS: Progress-free survival; PNI: Prognostic nutritional index; GPS: Glasgow prognostic score; AGR: Albuminto-globulin ratio; PRISMA: Preferred reporting items for systemic reviews and meta-analysis; NOS: Newcastle-Ottawa Scale.
\end{abstract}

\section{Acknowledgements}

We would like to thank the participants for their contributions.

\section{Authors' contributions}

XHN, JB: protocol/project development; XHN, ZZ: data collection; XHN: data analysis; XHN, ZZ: manuscript writing/editing. All authors read and approved the final manuscript.

\section{Funding}

Fund from Shanghai Public Health Clinical Center (KY-GW-2018-46).

\section{Availability of data and materials}

The information used and analyzed during this study is available from the original literature listed in the reference. The analysis methods can be obtained from the corresponding author upon reasonable request.

\section{Ethics approval and consent to participate}

Not applicable.

\section{Consent for publication}

Not applicable.

\section{Competing interests}

There are no conflicts of interest for any author.

Received: 1 December 2020 Accepted: 6 February 2021

Published online: 19 February 2021

\section{References}

1. Torre LA, Bray F, Siegel RL, Ferlay J, Lortet-Tieulent J, Jemal A. Global cancer statistics, 2012. CA Cancer J Clin. 2015;65:87-108.

2. Siegel RL, Miller KD, Jemal A. Cancer statistics, 2019. CA Cancer J Clin. 2019;69:7-34

3. Siegel RL, Miller KD, Jemal A. Cancer statistics, 2020. CA Cancer J Clin. 2020;70:7-30.

4. Bray F, Ferlay J, Soerjomataram I, Siegel RL, Torre LA, Jemal A. Global cancer statistics 2018: GLOBOCAN estimates of incidence and mortality worldwide for 36 cancers in 185 countries. CA Cancer J Clin. 2018:68:394-424.

5. Jerónimo C, Henrique R. Epigenetic biomarkers in urological tumors: A systematic review. Cancer Lett. 2014;342:264-74.

6. Rouprêt M, Babjuk M, Compérat E, et al. European Association of Urology Guidelines on Upper Urinary Tract Urothelial Carcinoma: 2017 Update. Eur Urol. 2018;73:111-22.
7. Mantzorou M, Koutelidakis A, Theocharis S, Giaginis C. Clinical Value of Nutritional Status in Cancer: What is its Impact and how it Affects Disease Progression and Prognosis? Nutr Cancer. 2017;69:1151-76.

8. Qi F, Zhou X, Wang Y, et al. Pre-treatment prognostic nutritional index may serve as a potential biomarker in urinary cancers: a systematic review and meta-analysis. Cancer Cell Int. 2018;18:207.

9. Qi F, Xu Y, Zheng Y, Li X, Gao Y. Pre-treatment Glasgow prognostic score and modified Glasgow prognostic score may be potential prognostic biomarkers in urological cancers: a systematic review and meta-analysis. Ann Transl Med. 2019;7:531.

10. Mori K, Miura N, Mostafaei $\mathrm{H}$, et al. Prognostic value of preoperative hematologic biomarkers in urothelial carcinoma of the bladder treated with radical cystectomy: a systematic review and meta-analysis. International Journal of Clinical Oncology. 2020;25:1459-74.

11. Liu J, Wang F, Li S, Huang W, Jia Y, Wei C. The prognostic significance of preoperative serum albumin in urothelial carcinoma: a systematic review and meta-analysis. Biosci Rep. 2018;38:12.

12. Takagi K, Domagala P, Polak WG, Buettner S, Wijnhoven BPL, ljzermans JNM. Prognostic significance of the controlling nutritional status (CONUT) score in patients undergoing gastrectomy for gastric cancer: a systematic review and meta-analysis. BMC Surg. 2019;19:129.

13. Takagi K, Domagala P, Polak WG, Buettner S, ljzermans JNM. Prognostic significance of the controlling nutritional status (CONUT) score in patients undergoing hepatectomy for hepatocellular carcinoma: a systematic review and meta-analysis. BMC Gastroenterol. 2019;19:211.

14. Takagi K, Buettner S, ljzermans JNM. Prognostic significance of the controlling nutritional status (CONUT) score in patients with colorectal cancer: A systematic review and meta-analysis. Int J Surg. 2020;78:91-6.

15. Miyake M, Morizawa Y, Hori S, et al. Integrative Assessment of Pretreatment Inflammation-, Nutrition-, and Muscle-Based Prognostic Markers in Patients with Muscle-Invasive Bladder Cancer Undergoing Radical Cystectomy. Oncology. 2017;93:259-69.

16. Takemura K, Yuasa T, Fujiwara R, et al. Prognostic Significance of the controlling nutritional status (CONUT) score in patients with advanced renal cell carcinoma treated with nivolumab after failure of prior tyrosine kinase inhibitors. J Urol. 2020;204:1166-72.

17. Bao Z, Li Y, Guan B, et al. High preoperative controlling nutritional status score predicts a poor prognosis in patients with localized upper tract urothelial cancer: a propensity score matching study in a large Chinese center. Cancer Manag Res. 2020;12:323-35.

18. Song $\mathrm{H}, \mathrm{Xu} \mathrm{B}$, Luo $\mathrm{C}$, et al. The prognostic value of preoperative controlling nutritional status score in non-metastatic renal cell carcinoma treated with surgery: a retrospective single-institution study. Cancer Manag Res. 2019;11:7567-75.

19. Moher D, Shamseer L, Clarke M, et al. Preferred reporting items for systematic review and meta-analysis protocols (PRISMA-P) 2015 statement. Syst Rev. 2015;4:1.

20. Stang A. Critical evaluation of the Newcastle-Ottawa scale for the assessment of the quality of nonrandomized studies in meta-analyses. Eur J Epidemiol. 2010;25:603-5.

21. Higgins JP, Thompson SG. Quantifying heterogeneity in a meta-analysis Stat Med. 2002;21:1539-58.

22. Guo K, Wang T, Yuan S, et al. Applicative value of controlling nutritional status to predict survival after cystectomy for patients with bladder cancer. J Mod Urol. 2017:22:925-9.

23. Zhang W, Wu Y, Zhang Z, et al. Controlling Nutritional Status score: a new prognostic indicator for patients with oligometastatic prostate cancer. Curr Probl Cancer. 2019;43:461-70.

24. Elghiaty A, Kim J, Jang WS, et al. Preoperative controlling nutritional status (CONUT) score as a novel immune-nutritional predictor of survival in non-metastatic clear cell renal cell carcinoma of $\leq 7 \mathrm{~cm}$ on preoperative imaging. J Cancer Res Clin Oncol. 2019;145:957-65.

25. Ishihara H, Kondo T, Yoshida K, et al. Preoperative controlling nutritional status (CONUT) score as a novel predictive biomarker of survival in patients with localized urothelial carcinoma of the upper urinary tract treated with radical nephroureterectomy. Urol Oncol. 2017;35:539.e9-. e16.

26. Kang HW, Seo SP, Kim WT, et al. Prognostic impact of nutritional status assessed by the controlling nutritional status (CONUT) score in patients with surgically treated renal cell carcinoma. Nutr Cancer. 2018;70:886-94. 
27. Zheng Y, Bao L, Wang W, Wang Q, Pan Y, Gao X. Prognostic impact of the controlling nutritional status score following curative nephrectomy for patients with renal cell carcinoma. Medicine. 2018;97:e13409.

28. Suzuki H, Ito M, Takemura K, et al. Prognostic significance of the controlling nutritional status (CONUT) score in advanced urothelial carcinoma patients. Urol Oncol. 2020;38:11-7.

29. $X u H, \operatorname{Tan} P$, Jin $X$, et al. Validation of the preoperative controlling nutritional status score as an independent predictor in a large Chinese cohort of patients with upper tract urothelial carcinoma. Cancer Med. 2018;7:6112-23.

30. Capitanio U, Bensalah K, Bex A, et al. Epidemiology of renal cell carcinoma. Eur Urol. 2019;75:74-84

31. Chamie K, Litwin MS, Bassett JC, et al. Recurrence of high-risk bladder cancer: a population-based analysis. Cancer. 2013;119:3219-27.

32. Fu XL, Duan W, Su CY, et al. Interleukin 6 induces M2 macrophage differentiation by STAT3 activation that correlates with gastric cancer progression. Cancer Immunol Immunother. 2017;66:1597-608.

33. Chimento A, Casaburi I, Avena P, et al. Cholesterol and its metabolites in tumor growth: therapeutic potential of statins in cancer treatment. Front Endocrinol (Lausanne). 2018:9:807.
34. Resnik N, Sepcic K, Plemenitas A, Windoffer R, Leube R, Veranic P. Desmosome assembly and cell-cell adhesion are membrane raft-dependent processes. J Biol Chem. 2011;286:1499-507.

35. Minami T, Minami T, Shimizu N, et al. Identification of programmed death ligand 1-derived peptides capable of inducing cancer-reactive cytotoxic T lymphocytes from HLA-A24 + patients with renal cell carcinoma. J Immunother. 2015;38:285-91.

36. Mehrazin R, Uzzo RG, Kutikov A, et al. Lymphopenia is an independent predictor of inferior outcome in papillary renal cell carcinoma. Urol Oncol 2015;33:388.e19-25

\section{Publisher's note}

Springer Nature remains neutral with regard to jurisdictional claims in published maps and institutional affiliations.
Ready to submit your research? Choose BMC and benefit from:

- fast, convenient online submission

- thorough peer review by experienced researchers in your field

- rapid publication on acceptance

- support for research data, including large and complex data types

- gold Open Access which fosters wider collaboration and increased citations

- maximum visibility for your research: over $100 \mathrm{M}$ website views per year

At BMC, research is always in progress.

Learn more biomedcentral.com/submissions 